\title{
The Parametric Definition of Membership Functions in XFL3
}

\author{
F. J. Moreno-Velo, I. Baturone, S. Sánchez-Solano , A. Barriga \\ Instituto de Microelectrónica de Sevilla - Centro Nacional de Microelectrónica \\ Avda. Reina Mercedes s/n, (Edif. CICA) \\ E-41012, Sevilla, Spain.
}

Proc. IEEE International Conference on Fuzzy Systems (FUZZIEEE 2004),

Budapest, July 25-29, 2004.

(C) 2004 IEEE. Personal use of this material is permitted. However, permission to reprint/republish this material for advertising or promotional purposes or for creating new collective works for resale or redistribution to servers or lists, or to reuse any copyrighted component of this work in other works must be obtained from the IEEE.

This material is presented to ensure timely dissemination of scholarly and technical work. Copyright and all rights therein are retained by authors or by other copyright holders. All persons copying this information are expected to adhere to the terms and constraints invoked by each author's copyright. In most cases, these works may not be reposted without the explicit permission of the copyright holder. 


\title{
The Parametric Definition of Membership Functions in XFL3
}

\author{
F. J. Moreno Velo, I. Baturone, S. Sánchez Solano, A. Barriga \\ Instituto de Microelectrónica de Sevilla (IMSE-CNM) \\ Centro Nacional de Microelectrónica - CSIC \\ Avda. Reina Mercedes, s/n. Edif. CICA, E-41012, Sevilla, Spain \\ E-mail: xfuzzy-team@imse.cnm.es
}

\begin{abstract}
This paper presents a study of the different kinds of membership function (MF) definitions, regarding free MFs and families of MFs, and describes the capabilities of XFL3 (the formal specification language defined by Xfuzzy 3) to manage them. This includes not only the possibility of using them in a system design, but also the capability for extending the available functions with new userdefined membership functions and families. An application example has been included in order to discuss on the suitable parametric definition of the functions.
\end{abstract}

\section{INTRODUCTION}

Membership functions, as the way of representing fuzzy sets, can be seen as the basis of any fuzzy methodology [1]. The linguistic meaning of fuzzy sets makes the most usual function shapes be: $\delta$-functions (corresponding to the fuzzification of crisp values), $\pi$-functions (intervals) and s-functions and z-functions (extreme intervals) [2], as shown in Fig. 1. Other kinds of membership functions can be obtained as a modification or aggregation of these basic forms.

From a practical point of view, membership functions are commonly expressed as analytical functions. The most frequently used analytical functions include piecewise linear functions (such as triangles, trapezoids or slopes) and non-linear functions (such as gaussians, bells or sigmoids) [3]. Fig. 2 shows a graphical representation of these functions. The mathematical expressions of these functions usually contain several parameters, which have to be valued when defining the membership function. For example, a triangular function

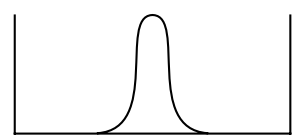

(a)

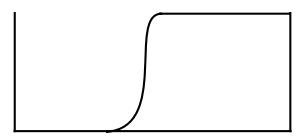

(c)

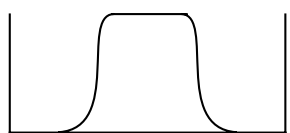

(b)

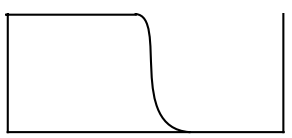

(d)
Fig. 1: The usual basic forms of membership functions

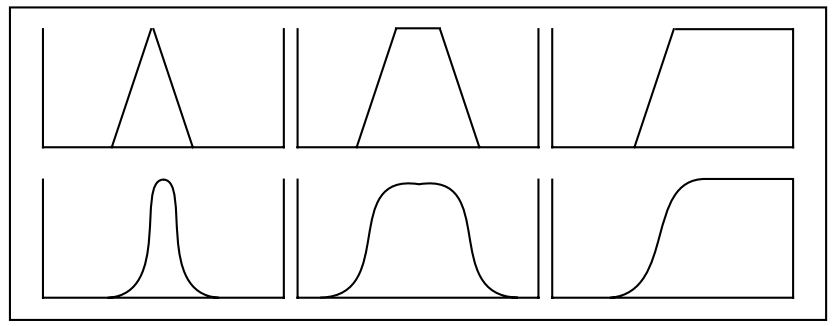

Fig. 2: Some of the most used analytical functions

can be defined by means of three parameters, representing either the apex and limit points of the triangle (Fig. 3.a), or its apex, width and slant (Fig. 3.b). Hence, the selection of these parameters is another degree of freedom in the design space of a fuzzy system.

Opposite to what happens to other degrees of freedom, it is important not only to consider the shape of the membership functions, but also the parameters used in their mathematical expressions when confronting the design of a fuzzy system. In general, the selection of the parametric expression of membership functions has not been much valued by the fuzzy system designers. The selection of other features, such as fuzzy operators, defuzzification methods or the shape of the membership functions have motivated several studies, as these aspects drive to differences in the system behavior. On the other hand, the definition of a membership function in terms of a set of parameters or other does not modify the system behavior, what explains the apparent lack of interest in this field.

When applying soft computing techniques in the different stages of fuzzy system design (such as the automatic identification, tuning or simplification of systems) the selection of the parametric expression of the membership functions play an important rule in the computer aided design. These techniques use to automatically modify the values of the parameters, and so, an adequate selection of these parameters may be used to maintain the linguistic interpretability of the final results of these stages.

This paper is structured as follows. Section II presents the different kinds of parametric membership functions defined and their related linguistic interpretation. Section III explains 


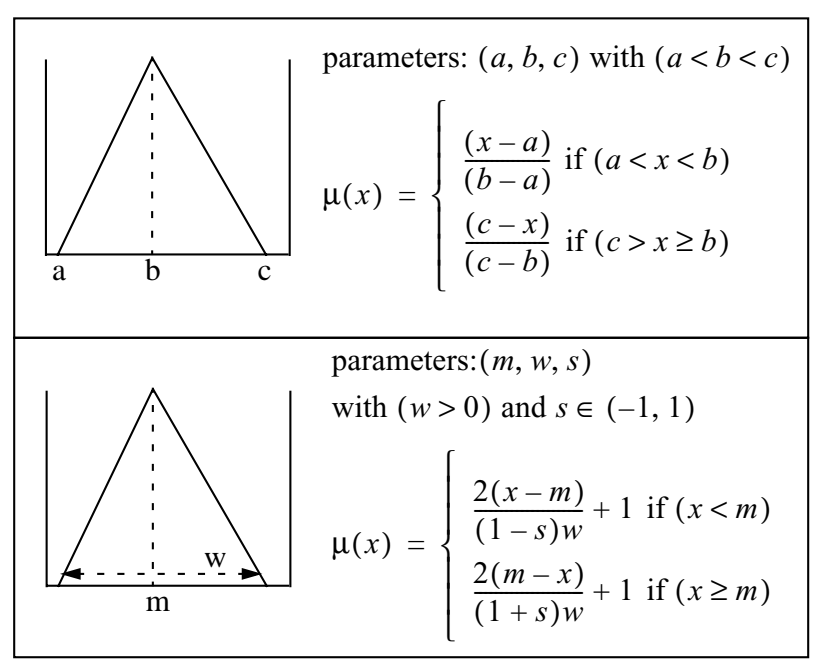

Fig. 3: Descriptions of a triangular membership function

how these kinds of definition have been implemented in XFL3, the formal specification language defined by the Xfuzzy 3 development environment. Finally, Section IV shows an application example in which these different membership function definitions have been used to solve a specific problem.

\section{THE DIFFERENT KINDS OF MF DEFINITIONS}

The first option when defining a set of membership functions (related with the linguistic labels of a variable) is whether to use a list of parameters to define the whole set of functions or to describe each membership function with its own and independent list of parameters. In the first case we will talk about families of membership functions while the second case is usually known as free membership functions (Fig. 4).

The definition of membership functions as families has several advantages. Firstly, the number of parameters to use is smaller than that of the free membership functions. This eases the tuning of the fuzzy system and permits the use of some automatic learning algorithms (such as simulated annealing or genetic algorithms) that are not appropriate with a large number of parameters. Secondly, the use of common list of parameters makes it ease to guarantee the linguistic meaning of the membership functions. In particular, the membership functions cannot evolve by construction to a state with highly overlapped or disordered functions in an automatic modification process. Finally, a family of membership functions can be defined with a fixed overlapping degree. This is very useful to simplify the inference mechanism (by using grid partitions), which is essential to confront the hardware synthesis of fuzzy systems. The main disadvantage of this kind of definition is that it imposes hard constraints on the functions, which could be an obstacle to a suitable system optimization (specially concerning the output variables descriptions).

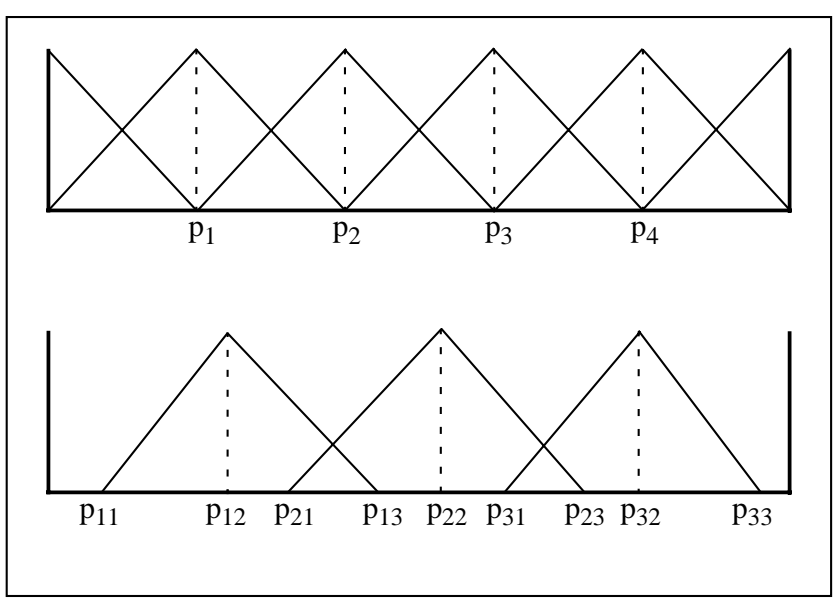

Fig. 4: Different kinds of MF definitions:

(a) Family of MFs; (b) Free MFs.

On the other hand, the definition of free membership functions does not impose any restriction when applying automatic modification processes so that the position, form and overlapping degree of the functions can be optimized, which could be very interesting in several knowledge acquisition problems. Another important advantage is that free membership functions get better results when optimizing fuzzy systems. The main disadvantages are that the linguistic meaning of the functions can be lost in the tuning process and that the final representation can be incompatible with simplified inference schemes.

In general, free membership functions are more appropriate to describe output variables, while families of membership functions are specially indicated to describe input variables. When there is not much information about the structure of an input variable, it can be useful to describe it by free membership functions and then to perform an automatic tuning process to acquire some knowledge on the form and overlapping degree of these functions. In a second step, this information can be used to describe the input variable in terms of a suitable family of membership functions.

An important issue, both for defining families of membership functions or free membership functions, is the selection of the most suitable parametric description. A suitable parametric description should allow a high degree of freedom capable of maintaining the linguistic meaning of the fuzzy sets as well as the particular constraints desired by the designer. Fig. 5 shows an example with a set of 7 triangular membership functions. If they are described as free membership functions, 21 parameters ( 3 per function) are required (Fig. $5 b$ ). It is possible to maintain the order of the functions, and also an overlapping degree of 2, using a family of membership functions with 5 parameters (Fig. $5 \mathrm{c}$ ). If the linguistic variable to describe is known to have a symmetrical behavior, it is more suitable to describe it with a family of symmetrical membership functions, using only 2 parameters (Fig. 5d).

The capability of selecting free membership functions as 


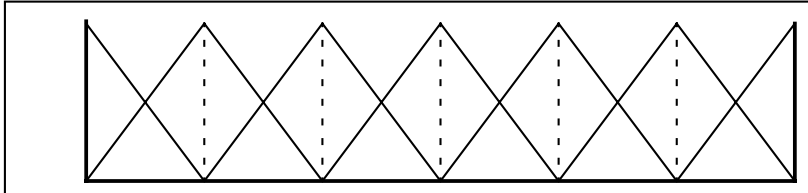

(a)

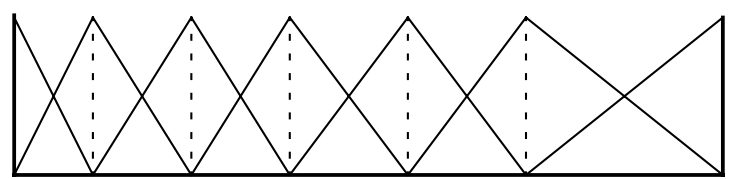

(c)

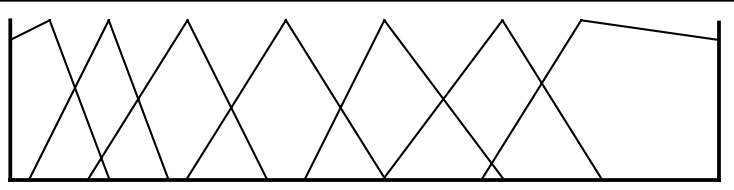

(b)

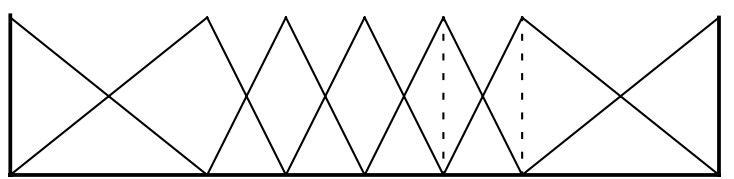

(d)

Fig. 5: Example of the evolution of a set of membership functions: (a) initial state; (b) possible final state with free MFs; (c) possible final state with a family of triangular MFs; (d) possible final state with a family of symmetric and triangular MFs.

well as families of membership functions is a very suitable feature for a CAD environment devoted to fuzzy system design. Such an environment should offer the most usual functions and families. A typical set of free membership functions contains triangles, trapezoids, gaussians, bells, sigmoids and singletons. On the other hand, it is worth considering set of families containing triangular, bell-shaped, trapezoid-triangular and B-splines with an overlapping degree of 3, as well as their symmetrical versions. Another desirable feature for these environments is the capability of extending the membership functions and families offered and the possibility of using these user-defined functions by automatic tuning processes. The next section presents how the development environment Xfuzzy 3 has supported these desirable characteristics.

\section{The MF DEFINITION IN XFL3}

XFL3 is a formal specification language for the description of complex fuzzy systems [4]. It has been defined as the common specification language of Xfuzzy 3, a development environment for fuzzy systems [5]. Xfuzzy 3 integrates several tools covering the different stages in the design flow: description, identification, tuning, simplification, verification and synthesis. The main features of XFL3 are a high expressiveness, extensiveness and modularity. Modularity refers to the capability of XFL3 for describing hierarchical systems with different modules: rule bases and crisp blocks. Expressiveness has been obtained by the definition of fuzzy rules in terms of any combination of fuzzy connectives and linguistic hedges. Finally, extensibility is supported by the inclusion of a mechanism to let the designer to create its own fuzzy operators, defuzzification methods, linguistic hedges, crisp blocks, free membership functions and families of membership functions, extending those available in the environment.

The description of a fuzzy system in XFL3 is divided into two parts: the logical description (included in files with extension "xfl"), and the functional description, that is, the mathematical description of the functions used in the system (included in files called function packages). The logical de- scription is composed of three kinds of objects (linguistic types, operator sets, and rule bases) and the definition of the hierarchical structure of the system. The definition of the membership functions assigned to a linguistic variable is included into a type object. The syntax of this object in XFL3 is the following:

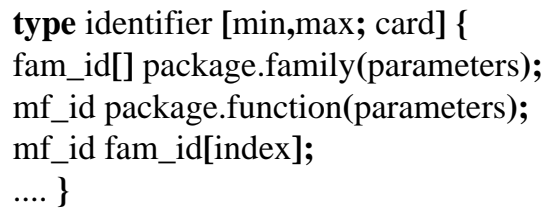

This definition includes the name of the type, the description of the universe of discourse ( $\min$ and max refer to the limits of the universe; card is the cardinality), the list of linguistic labels ( $m f \_i d$ ), and their related membership functions. A membership function can be a free membership function (selected from a function package) or a member of a family of membership functions. In the last case, the family has to be previously selected from a function package. Figure 6 shows an example with two type definitions, one of them based on free functions and the other using a family of functions.

Function packages include the mathematical definition of those functions used in the logical description of fuzzy systems. These packages are defined in files with extension "pkg" and can be freely created, as a mechanism to extend the available functions for the system development. A package contains a set of function definitions, including binary functions (related with fuzzy connectives), unary functions (related with linguistic hedges), defuzzification methods, crisp functions, free membership functions and families of membership functions. These definitions are based on code blocks describing the function parameters, the mathematical description of the function in several programming languages, and some other features of the functions.

Figure 7 and Figure 8 show an example of the definition of a free membership function and the definition of a family of membership functions, respectively. These examples illus- 


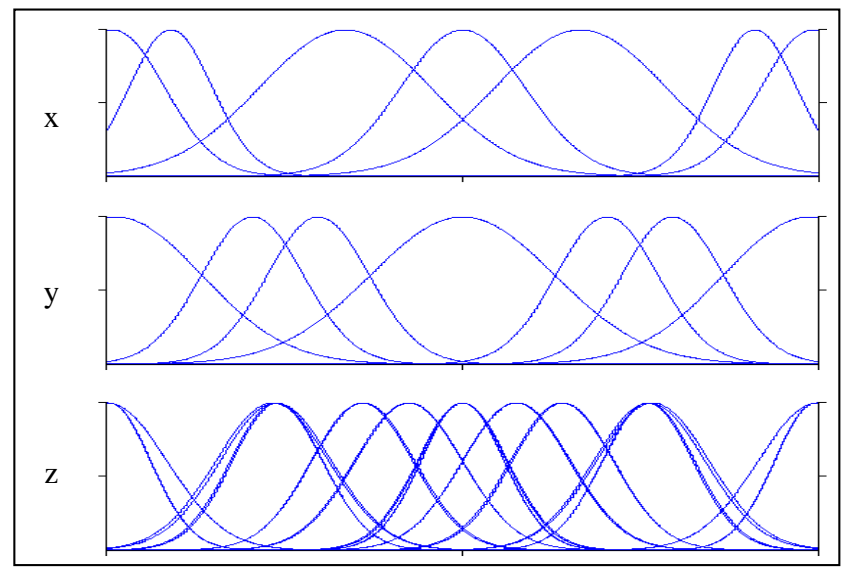

Fig. 10: Representation of variables using free MFs

overlapping degree seems to be limited to 3 . Using this information, a new fuzzy system can be created describing the input variables in terms of a family of symmetrical B-splines with a fixed overlapping degree of 3 . The number of parameters for the input variables is reduced from 28 to 4 , as each input variable can be defined in terms of only 2 parameters. Figure 11 shows a graphical representation of the variables after a new tuning process. As in the previous case, the output membership functions trend to bunch up around certain values, so their number has been reduced form 49 to 9 by applying a clustering process. The RMSE of the new system is $0.89 \%$. The use of families of membership functions has increased the system deviation a little, but now the order and symmetry of the membership functions have been fixed by construction. It is also worth considering that the active rules are limited now to 9 instead of 49 . This fact can be exploited to increase the inference speed and to reduce the complexity of fuzzy systems implemented in specific hardware or embedded software (by applying active-rule-based inference mechanisms).

\section{CONCLUSIONS}

When including automatic tuning techniques into the design flow of fuzzy systems, the parametric description of membership functions becomes very important. A suitable parametric description allows maintaining some important features of the functions like order, symmetry or overlapping degree. The last version of XFL3 (the formal language of Xfuzzy 3) supports both free membership functions and families of membership functions, which can be freely extended by the designer. Xfuzzy 3 is platform independent (programmed in Java) and it is distributed under the GNU General Public license. More detailed information on XFL3 and Xfuzzy 3 can be found at the official Xfuzzy site:

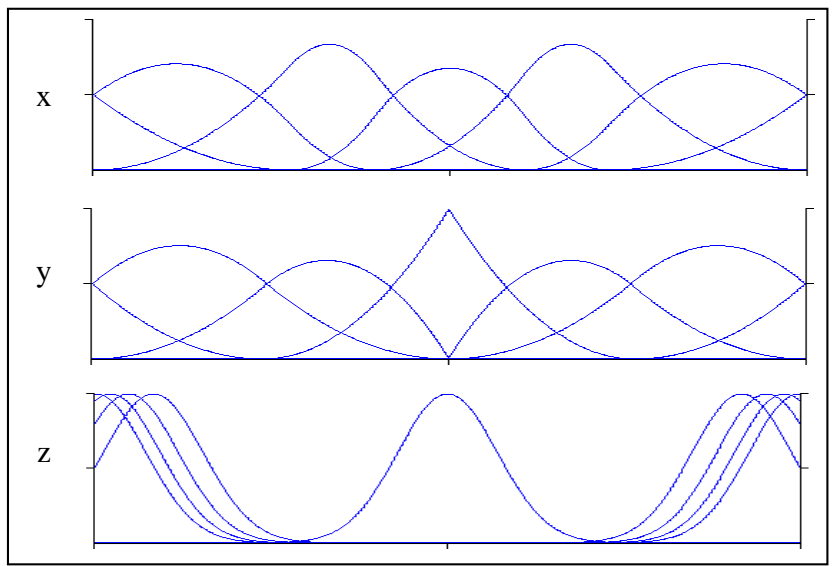

Fig. 11: Representation of variables using families of MFs

\section{ACKNOWLEDGMENT}

This work has been partially supported by the Spanish CICYT Project TIC2001-1726.

\section{REFERENCES}

[1] L.A. Zadeh, "Fuzzy sets", Information and control, Vol.8, pp. 338-358, 1965.

[2] E. Cox, The Fuzzy Systems Handbook, AP Professional, 1999.

[3] T.J. Ross, Fuzzy Logic with Engineering Applications, McGraw-Hill inc., 1995.

[4] F.J. Moreno-Velo, S. Sánchez-Solano, A. Barriga, I. Baturone, D. R. López, "XFL3: A New Fuzzy System Specification Language", Mathware \& Soft Computing, Vol. 8, No. 3, pp. 239- 253, 2001.

[5] F.J. Moreno Velo, I. Baturone, S. Sánchez Solano, A. Barriga, "Rapid Design of Fuzzy Systems with Xfuzzy", IEEE Int. Conf. on Fuzzy Systems (FUZZ-IEEE 2003), pp. 342-347.

[6] R. Rovatti, R. Guerrieri, "Fuzzy sets of rules for system identification”, IEEE Trans. Fuzzy Systems, Vol 4, No. 2, pp.89-102, 1996.

[7] F.J. Moreno Velo, I. Baturone, R. Senhadji, S. Sánchez Solano, "Tuning Complex Fuzzy Systems by Supervised Learning Algorithms", IEEE Int. Conf. on Fuzzy Systems (FUZZ-IEEE 2003), pp. 226-231. 